\title{
Paying Attention to What Works in Therapy: The Case for Feedback Informed Treatment
}

\author{
Klosterman $\mathrm{K}^{1,2 *}$, Papagni $\mathrm{E}^{1}$ and Mignone $\mathrm{T}^{3}$ \\ ${ }^{1}$ Medaille College, USA \\ ${ }^{2}$ Wheatfield Pediatrics, USA \\ ${ }^{3}$ Enlightened Therapies of Western New York, USA
}

*Corresponding author: Keith Klostermann, Medaille College, 18 Agassiz Circle,

Mini Review

Volume 3 Issue 2

Received Date: September 30, 2019

Published Date: October 15, 2019

DOI: $10.23880 /$ mhrij-16000134

Buffalo, USA, Email: kck35@medaille.edu.

\section{Abstract}

It is critical that therapists identify when therapy is not working and collaborate with clients on how treatment should be modified, according to their preferences. One method for routinely monitoring outcomes involves the use of Feedback Informed Treatment. FIT is based on 2 primary findings: 1) Clients subjective experience of the therapist in treatment (i.e., view of the alliance) is one of the best predictors of outcome and 2) Clients experience of change early in treatment is a very good predictor of treatment outcome. FIT involves the use of two psychometrically sound and brief measures: 1) Outcome Rating Scale (ORS), and 2) Session Rating Scale (SRS). Implementing and practicing FIT is about more than just collecting feedback and may involve developing a closer relationship with research, statistics, and measurement. Paying Attention to What Works in Therapy: The Case for Feedback Informed Treatment.

Keywords: Therapy; Clients; Feedback Informed Treatment

Abbreviations: ORS: Outcome Rating Scale; SRS: Session Rating Scale; FIT: Feedback Informed Treatment.

\section{Mini Review}

Although an extensive literature has evolved regarding the importance of the therapeutic relationship as a necessary component of treatment, it is not sufficient inand-of-itself; therapists must also pay attention to outcome and whether what they are doing is working for their clients. While it's easy to tell when therapy works, it is less obvious to identify when it's not working. In fact, dropout rates range from 25 to $46 \%$ [1]. Therefore, it is critical that we are aware when therapy is not working and collaborate with clients on how treatment should be modified, according to their preferences. In other words, it is imperative that therapists recognize when therapy isn't working and negotiate alternatives.

\section{Feedback Informed Treatment (Fit)}

Measuring constructs previously found to be associated with successful outcomes can be utilized to promote change as well as identify cases which are off 


\section{Mental Health \& Human Resilience International Journal}

track and/or at risk of dropout or failure. One method for routinely monitoring outcomes involves the use of Feedback Informed Treatment [2]. FIT is based on 2 primary findings: 1) Clients subjective experience of the therapist in treatment (i.e., view of the alliance) is one of the best predictors of outcome and 2) Clients experience of change early in treatment is a very good predictor of treatment outcome. Simply stated, asking clients about their perception of the alliance provides a critical opportunity for therapists to change what they're doing to better accommodate what clients' want, the way they want it, delivered to their preferences. In addition, numerous research studies suggest that, on average, the bulk of change in successful therapy occurs earlier rather than later [3]. Simply put, early change is predictive of more robust change later on Howard. Consequently, the longer therapy lasts, working in a particular way, with a particular client, at a particular time, in that location, without a change, the greater the chances that this particular client-therapist relationship will end in either dropout or the client continues the course of treatment but doesn't benefit from the services offered.

Consistent with these findings, clients' perception of the alliance as well as early change in treatment form the basis of FIT. Given these two findings, it is critical that therapists identify whether the client feels engaged and if not, adjust the treatment to better fit the client's needs as way to improve retention and decrease dropouts.

FIT is pantheoretical and can be applied to any discipline (psychiatry, social work, psychology) or approach (e.g., CBT, solution focused, family therapy). The recent emphasis in the field on continuous quality improvement, plus the move toward patient focused research, has created a climate for practice-based evidence in which treatment is driven by the client's perception of progress. FIT involves the use of two psychometrically sound and brief measures: 1) Outcome Rating Scale (ORS), and 2) Session Rating Scale (SRS). Each measure consists of four items and takes less than a minute to complete; measures that require more than 5 minutes to complete, score, and interpret are not feasible [4]. To be clear, feedback informed treatment means therapists are asking for their client's perception in a much more formal way than verbally inquiring about the work being doing. Reasons for using FIT include: 1) improves outcomes one client at a time, 2) identifies areas for performance improvement, 3) identifies cases at risk of getting worse or even dropout, and 4) helps reduce variability in performance between clinicians. According to Hawkins, et al. [5], collecting feedback and engaging clients in discussion improves outcomes, and alerts clinicians to the possibility of failure. Sadly, despite the availability and accessibility of outcome measures, few therapists actually use them in their clinical practice [6]. Further, many practitioners who measure progress at every session do not use the feedback to inform the treatment process. De Jong found that therapists could not be counted on to ask for feedback or use it productively when provided.

\section{Barriers to FIT}

There are several barriers to fit, for example, therapists think they are already doing it, therapists believe that it is inconsistent with their preferred way of working, and therapists who are used to moving speedily into what they believe are the primary components of a treatment model or goals may be concerned that FIT will slow the pace of treatment. Furthermore, some therapists are unfamiliar with the culture of feedback, which involves several variables such as taking time to introduce the outcome and alliance measures to clients in a thoughtful manner, providing a clear rationale as well as explaining how the feedback will be used to guide treatment. Moreover, it is crucial that that the client understands that the therapist is not going to be offended or become defensive when given feedback.

Implementing and practicing FIT is about more than just collecting feedback and may involve developing a closer relationship with research, statistics, and measurement [2]. It also means that therapists need to feel comfortable making themselves vulnerable to their clients' perceptions of progress and a display a willingness to use client feedback to drive the therapy process. Unfortunately, for some, there is an unspoken expectation that the client must change according to a process set by the therapist [7]. Yet, current research indicates that clinician's self-assessment is not reliable (tend to overestimate their effectiveness) and clinicians are rarely accurate nor reliable in identifying clients who are getting worse in care [8]. The most effective clinicians consistently assess both progress and alliance at each session.

\section{References}

1. Wierzbicki M, Pekarik G (1993) A meta-analysis of psychotherapy dropout. Professional Psychology: Research and Practice 24(2): 190-195. 


\section{Mental Health \& Human Resilience International Journal}

2. Miller SD (2011) Psychometrics of the ORS and SRS. Results from RCTs and Meta-analyses of Routine Outcome Monitoring \& Feedback. The Available Evidence, Chicago.

3. Lambert MJ, Ogles BM (2004) The efficacy and effectiveness of psychotherapy. In: Lambert MJ, (Eds.), Bergin and Garfield's handbook of psychotherapy and behavior change, $5^{\text {th }}$ (Edn.), New York, pp: 139-193.

4. Brown J, Dreis S, Nace DK (1999) What really makes a difference in psycotherapy outcome? Why Does Managed Care Want to Know? In: Hubble MA, Ducan SD, Miller D, (Eds.), The Heart and Soul of Change: What Works in Therapy, American Psychological Association, Washington DC, pp: 389-406.
5. Hawkins EJ, Lambert MJ, Vermeesch, DA, Slade KL, Tuttle KC (2004). The therapeutic effects of providing patient progress information to patients and therapists. Psychotherapy Research 14(3): 308-327.

6. Zimmerman M, McGlinchey JB (2008) Why don't psychiatrists use scales to measure outcome when treating depressed patients? Journal of Clinical Psychiatry 69(12): 1916-1919.

7. Miller SD, Duncan BL, Hubble MA (2004) Beyond integration: The triumph of outcome over process in clinical practice. Psychotherapy in Australia 10: 3243.

8. Miller SD, Hubble MA, Chow DL, Seidel JA (2013) The outcome of psychotherapy: Yesterday, today, and tomorrow. Psychotherapy 50(1): 88-97. 